\title{
Incidence and Characteristics of Scarlet Fever, South Korea, 2008-2015
}

\author{
Duck Woong Park, Sun-Hee Kim, \\ Jung Wook Park, Min-Ji Kim, Sun Ju Cho, \\ Hye Jung Park, So Hyang Jung, Mi Hee Seo, \\ Yong Seok Lee, Byung Hee Kim, Hyeran Min, \\ Su Ya Lee, Dong Ryong Ha, Eun Sun Kim, \\ Yeongjin Hong, ${ }^{1}$ Jae Keun Chung ${ }^{1}$
}

The incidence rate for scarlet fever in South Korea is rising. During 2008-2015, we collected group A Streptococcus isolates and performed emm and exotoxin genotyping and disk-diffusion antimicrobial tests. Scarlet fever in South Korea was most closely associated with emm types emm4, emm28, emm1, and emm3. In 2015, tetracycline resistance started increasing.

Carlet fever is a common disease caused by group A Streptococcus (GAS; also known as Streptococcus pyogenes). In the Far East and the United Kingdom, the incidence of scarlet fever has been increasing since 2008 (1-3), and according to the Infectious Disease Statistics System of Korea, the incidence rate for scarlet fever in South Korea increased from 0.3 cases/100,000 persons in 2008 to 13.7 cases/100,000 persons in 2015 (https://is.cdc.go.kr/dstat/ index.jsp).

Several antimicrobial drugs, including $\beta$-lactams and tetracyclines, effectively treat scarlet fever, and macrolides and lincosamides can be used in patients with penicillin ( $\beta$-lactam) allergy $(4,5)$. However, resistance to erythromycin and clindamycin has been reported for GAS isolates in mainland China and Hong Kong, China $(2,3)$. The streptococcal $\mathrm{M}$ protein and exotoxins are 2 of several virulence factors in GAS (6). The streptococcal M protein is a long fimbrial adhesion protein encoded by $>220 \mathrm{M}$ protein gene sequence types (emm types). Because of the high genetic variability of emm, which varies by geographic region, molecular emm genotyping is mandatory for epidemiologic

Author affiliations: Health and Environment Research Institute of Gwangju, Gwangju, South Korea (D.W. Park, S.-H. Kim, J.W. Park, M.-J. Kim, S.J. Cho, H.J. Park, S.H. Jung, M.H. Seo, Y.S. Lee, D.R. Ha, E.S. Kim, J.K. Chung); Mirae Children's Hospital (NamGu), Gwangju (B.H. Kim); Jungang Children's Hospital, Gwangju (H. Min); Yesarang Pediatric Clinic, Gwangju (S.Y. Lee); Chonnam National University Medical School, Gwangju (D.W. Park, Y. Hong)

DOI: http://dx.doi.org/10.3201/eid2304.160773 investigations of GAS infections (7). The incidence of these infections is closely related to variations in the predominance of certain emm types (7). speA and speC, which are 2 of 11 genes encoding for superantigens found in GAS, are often associated with scarlet fever (8). Our objective was to identify the overall trend in the annual incidence and characteristics of scarlet fever in South Korea by studying its upsurge in Gwangju, South Korea, because incidence during the past 8 years was highest for this city (https:// is.cdc.go.kr/dstat/index.jsp).

\section{The Study}

The incidence of scarlet fever in the Gwangju metropolitan area is the highest among all South Korea cities (61.5 cases/100,000 persons); according to the Korean Disease Web Statistics System, the national incidence from 2008 through 2015 was 36.9 cases/100,000 persons (https:// is.cdc.go.kr/dstat/index.jsp). Incidence of scarlet fever in South Korea began to increase in 2011 (Figure 1, panel A), coinciding with an outbreak of scarlet fever in China and Hong Kong. Scarlet fever mainly occurs during the late fall, winter, and early spring. Our study results indicate that the incidence of scarlet fever in South Korea peaks in the winter; however, it also peaked in the summers of 2011 and 2015 (Figure 1, panel B).

During 2008-2015, we collected 1,460 pharyngeal swab samples from patients suspected of having scarlet fever from 8 major hospitals in the Gwangju metropolitan area. We tested the $\beta$-hemolytic isolates for susceptibility to bacitracin $(0.04 \mathrm{U})$ and for streptococcal grouping; a total of 705 samples were positive for GAS.

Because variation in the circulating emm types could contribute to the incidence of disease and changes in the epidemiology of scarlet fever, we determined the emm sequence type for the 705 samples by using a standard protocol (https://www.cdc.gov/streplab/protocol-emm-type.html) (2). A total of 11 different emm sequence types were identified (Figure 2, panels A and B). emm 4 (35.6\%, 251/705) was the most predominant. The other 3 predominant emm types were emm 28 (14.8\%, 104/705), emm 1 (14.5\%, 102/705), and emm $3(11.6 \%, 82 / 705)$, and these $4 \mathrm{emm}$ types accounted for $\approx 76.5 \%(539 / 705)$ of all isolates. These results differed from those of previous studies in mainland China and Hong Kong, where the outbreaks of scarlet fever were caused mainly by emm $12(2,3)$. Our results showed that

${ }^{1}$ These authors contributed equally to this article. 
emm3 in 2011 (the year incidence began increasing in South Korea) and emm 1 and emm 28 in 2015 (the year of a sharp increase in incidence) played major roles in the epidemics in the Gwangju metropolitan area (Figure 2, panel A). To our knowledge, emm 3 has not been reported to be prevalent in other Asian countries but has been associated with scarlet fever in the United Kingdom (9).

Scarlet fever is a toxin-mediated disease (10). Therefore, we tested all isolates for the presence of the speA and speC genes by using PCR and previously reported primer pairs and reaction conditions $(8)$. We found isolates that harbored speA $(57 / 705,8.1 \%)$, speC $(249 / 705,35.3 \%)$, and both $(3 / 705,0.4 \%)$. The exotoxin gene detection rate differed by $\mathrm{emm}$ gene type. The isolates positive for speA were predominantly emm $1(56.1 \%, 32 / 57)$ and emm 28 $(28.1 \%, 16 / 57)$; the other emm types made up only $15.8 \%$ (9/57). When we examined the reverse association, $31.4 \%$ (32/102) of emm 1 isolates and $15.4 \%(16 / 104)$ of emm 28 isolates were positive for speA; in contrast, only $1.8 \%$ (9/499) of the other emm types were positive for speA. The
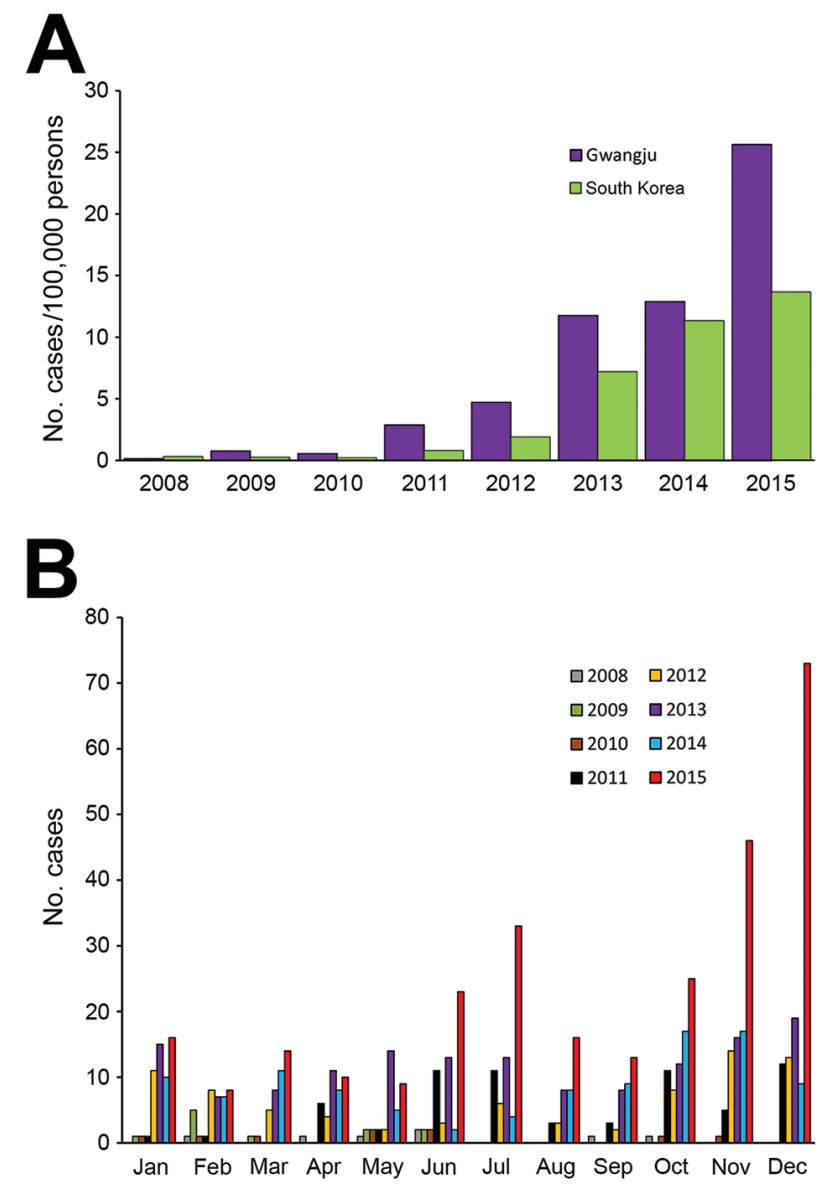

Figure 1. Incidence of scarlet fever in Gwangju, South Korea, 2008-2015. A) The number of cases per 100,000 persons in Gwangju and South Korea. B) Distribution of cases by month of each year. main emm types identified in the speC-positive isolates were emm $4(68.7 \%, 171 / 249)$, emm $75(10.8 \%, 27 / 249)$, and emm $28(6.4 \%, 16 / 249) ; 68.1 \%(171 / 251)$ of emm 4 , $67.5 \%(27 / 40)$ of emm 75 , and $15.4 \%(16 / 104)$ of emm 28 isolates were positive for speC. Only $11.3 \%(35 / 310)$ of the other emm types were positive for speC. Therefore, speA and $s p e C$ exotoxin genes were more prevalent in bacteria of certain emm types $(\mathrm{p}<0.01)$.
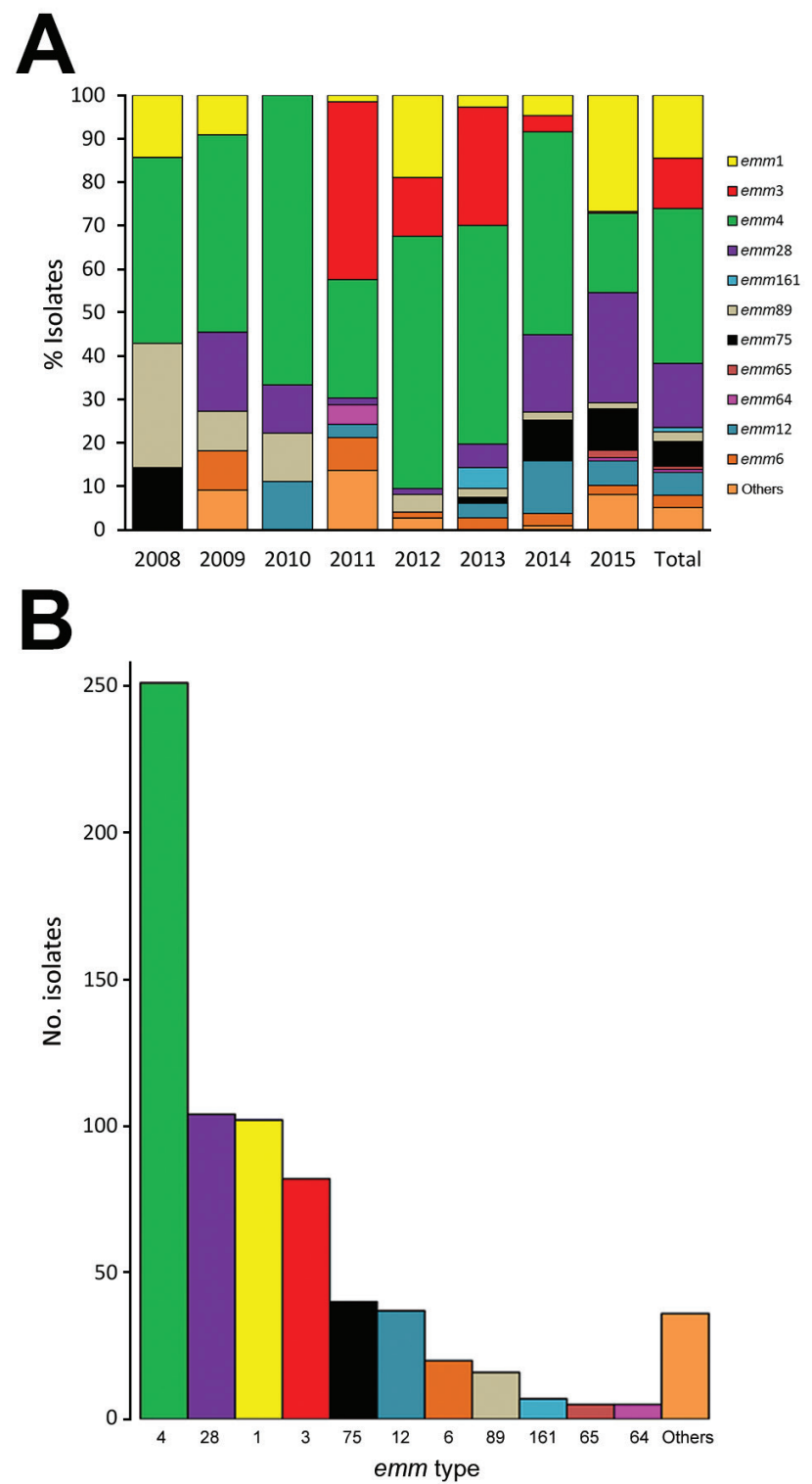

Figure 2. emm type characterization of group A Streptococcus isolates from patients with scarlet fever, Gwangju, South Korea, 2008-2015. A) Annual fluctuations of emm types. Number of isolates by year: 7 in 2008, 11 in 2009, 9 in 2010, 66 in 2011, 74 in 2012, 147 in 2013, 107 in 2014, and 284 in 2015. B) Total number of isolates by emm type. Others refers to rarely found emm types (emm11, emm13, emm17, emm23, emm26, emm30, emm31, emm43, emm49, emm59, emm81, emm82, emm87, emm101, emm107, emm131, emm135, emm161, emm163, emm174, emm183, emm196, emm203, emm204, emm227, emm236, and emm241). 
GAS remains universally susceptible to $\beta$-lactams and glycopeptides. However, the rates of resistance against the macrolides and lincosamides used in penicillin-allergic patients have increased (11). We performed susceptibility tests by using the disk-diffusion method as recommended by the Clinical and Laboratory Standards Institute (12). For all samples collected 2008-2015, the antimicrobial agents chloramphenicol, tetracycline, erythromycin, and clindamycin were tested. Resistance to antimicrobial drugs was detected in $9.1 \%(64 / 705)$ of isolates: $0.3 \%$ of the isolates $(2 / 705)$ showed resistance to chloramphenicol, $7.0 \%(49 / 705)$ to tetracycline, $3.0 \%(21 / 705)$ to erythromycin, and $2.8 \%(20 / 705)$ to clindamycin (Table).

In some isolates, antimicrobial drug resistance is tightly correlated with specific emm types (13). Resistance to erythromycin, clindamycin, and tetracycline is common in bacteria with the emm 28 gene. In our study, 18/104 $(17.3 \%)$ isolates that harbored emm28 were resistant to erythromycin, clindamycin, or tetracycline $(\mathrm{p}<0.01)$. Of all emm types, emm 28 accounted for $71.0 \%$ (44/62) of all cases of resistance to these 3 antimicrobial drugs. In our study, 16/44 isolates harboring emm 28 showed resistance to $\geq 2$ antimicrobials. emm 28 isolates in France were also found to be associated with multidrug resistance $(13,14)$. Furthermore, in 2015, we found sharp increases in intermediate tetracycline resistance mainly in isolates harboring emm $1(57.9 \%, 22 / 38)$. Tetracycline resistance associated with emm 12 and emm 1 isolates was also found in scarlet fever patients in Hong Kong and China $(3,15)$.

This study has a limitation. We collected samples from only 1 city in South Korea, the Gwangju metropolitan area. Because of the genetic diversity of GAS, our results should not be applied to other countries, even those nearby. However, we do believe that our data are representative of South Korea.

\section{Conclusions}

In 2011, rapid increases in the incidence of scarlet fever in South Korea, as well as China and Hong Kong, reflected the beginning of a pandemic in Asia. However, the emm types contributing to disease differed from country to country. emm 4 , emm 28 , emm 1 , and emm 3 were the most common

\begin{tabular}{|c|c|c|c|c|c|c|}
\hline \multirow[b]{2}{*}{ Year, antimicrobial drug } & \multicolumn{6}{|c|}{ emm type } \\
\hline & emm1 & emm4 & emm12 & emm28 & Others & Total \\
\hline \multicolumn{7}{|l|}{2008} \\
\hline Tetracycline & - & $1 \dagger$ & - & - & - & $1 \dagger$ \\
\hline \multicolumn{7}{|l|}{2009} \\
\hline Erythromycin & - & - & - & 2 & - & 2 \\
\hline Clindamycin & - & - & - & 2 & - & 2 \\
\hline Tetracycline & - & - & - & 1 & - & 1 \\
\hline \multicolumn{7}{|l|}{2010} \\
\hline Erythromycin & - & - & - & 1 & - & 1 \\
\hline Clindamycin & - & - & - & 1 & - & 1 \\
\hline \multicolumn{7}{|l|}{2011} \\
\hline Erythromycin & - & 1 & - & 1 & $1 \dagger$ & $1 / 3 \dagger$ \\
\hline Clindamycin & - & 1 & - & 1 & - & 2 \\
\hline Tetracycline & - & - & - & 1 & - & 1 \\
\hline \multicolumn{7}{|l|}{2012} \\
\hline Erythromycin & - & - & - & 1 & - & 1 \\
\hline Clindamycin & - & 1 & - & 1 & - & 2 \\
\hline Tetracycline & - & 1 & - & - & - & 1 \\
\hline \multicolumn{7}{|l|}{2013} \\
\hline Chloramphenicol & 1 & - & - & - & - & 1 \\
\hline Erythromycin & 1 & - & - & 2 & $1 \dagger$ & $1 / 4 \dagger$ \\
\hline Clindamycin & - & 1 & - & 2 & - & 3 \\
\hline Tetracycline & - & 1 & - & 1 & - & 2 \\
\hline \multicolumn{7}{|l|}{2014} \\
\hline Chloramphenicol & - & - & $1 \dagger$ & - & - & $1 \dagger$ \\
\hline Erythromycin & - & - & - & 4 & - & 4 \\
\hline Clindamycin & - & - & - & 4 & - & 4 \\
\hline Tetracycline & - & $1 \dagger$ & - & 4 & - & $1 / 5 \dagger$ \\
\hline \multicolumn{7}{|l|}{2015} \\
\hline Erythromycin & - & - & 1 & 5 & - & 6 \\
\hline Clindamycin & - & - & 1 & 5 & - & 6 \\
\hline Tetracycline & $22 / 23 \dagger$ & - & 1 & $2 / 7 \dagger$ & $5 / 7 \dagger$ & $29 / 38+$ \\
\hline $\begin{array}{l}\text { Isolates, \% (no./total) } \\
\text { p value }\end{array}$ & $\begin{array}{c}24.5 \%(25 / 102) \\
<0.01\end{array}$ & $\begin{array}{c}3.2 \%(8 / 251) \\
<0.01\end{array}$ & $10.8 \%(4 / 37)$ & $\begin{array}{c}17.3 \%(18 / 104) \ddagger \\
<0.01\end{array}$ & $4.3 \%(9 / 211)$ & $9.1 \%(64 / 705)$ \\
\hline
\end{tabular}


emm types associated with scarlet fever in South Korea. Antimicrobial drug resistance in GAS in South Korea is closely associated with emm 28 , and resistance to tetracycline (observed emerging in 2015) is associated with type $\mathrm{emm} 1$. However, further studies are necessary to characterize the circulating strains and to control and prevent the further spread of scarlet fever.

This study was supported by the infectious disease surveillance project from the Health and Environment Research Institute of Gwangju and the Korea National Institute of Health, South Korea.

Mr. D.W. Park works as a researcher in the Health and Environment Research Institute of Gwangju, Gwangju, South Korea. He is a doctoral candidate studying medicine at Chonnam National University Medical School, Gwangju, South Korea. His primary research interests are human infectious diseases, especially respiratory diseases.

\section{References}

1. Guy R, Williams C, Irvine N, Reynolds A, Coelho J, Saliba V, et al. Increase in scarlet fever notifications in the United Kingdom, 2013/2014. Euro Surveill. 2014;19:20749. http://dx.doi.org/10.2807/1560-7917.ES2014.19.12.20749

2. Lau EH, Nishiura H, Cowling BJ, Ip DKM, Wu JT. Scarlet fever outbreak, Hong Kong, 2011. Emerg Infect Dis. 2012;18:1700-2. http://dx.doi.org/10.3201/eid1810.120062

3. Yang P, Peng X, Zhang D, Wu S, Liu Y, Cui S, et al. Characteristics of group A Streptococcus strains circulating during scarlet fever epidemic, Beijing, China, 2011. Emerg Infect Dis. 2013;19:909-15. http://dx.doi.org/10.3201/eid1906.121020

4. Cunningham MW. Pathogenesis of group A streptococcal infections. Clin Microbiol Rev. 2000;13:470-511. http://dx.doi.org/10.1128/CMR.13.3.470-511.2000

5. Choi JH, Yang NR, Lee WJ, Lee H, Choi EH, Lee HJ. Distribution of emm types among group A Streptococcus isolates from children in Korea. Diagn Microbiol Infect Dis. 2015;82:26-31. http://dx.doi.org/10.1016/j.diagmicrobio.2015.01.002

6. Bisno AL, Brito MO, Collins CM. Molecular basis of group A streptococcal virulence. Lancet Infect Dis. 2003;3:191-200. http://dx.doi.org/10.1016/S1473-3099(03)00576-0
7. Steer AC, Law I, Matatolu L, Beall BW, Carapetis JR. Global emm type distribution of group A streptococci: systematic review and implications for vaccine development. Lancet Infect Dis. 2009;9:611-6. http://dx.doi.org/10.1016/S1473-3099(09)70178-1

8. Yamaoka J, Nakamura E, Takeda Y, Imamura S, Minato N. Mutational analysis of superantigen activity responsible for the induction of skin erythema by streptococcal pyrogenic exotoxin C. Infect Immun. 1998;66:5020-6.

9. Turner CE, Pyzio M, Song B, Lamagni T, Meltzer M, Chow JY, et al. Scarlet fever upsurge in England and molecular-genetic analysis in North-West London, 2014. Emerg Infect Dis. 2016;22:1075-8. http://dx.doi.org/10.3201/eid2206.151726

10. Silva-Costa C, Carriço JA, Ramirez M, Melo-Cristino J. Scarlet fever is caused by a limited number of Streptococcus pyogenes lineages and is associated with the exotoxin genes ssa, speA and speC. Pediatr Infect Dis J. 2014;33:306-10. http://dx.doi.org/10.1097/INF.0000000000000088

11. Plainvert C, Doloy A, Loubinoux J, Lepoutre A, Collobert G, Touak G, et al.; CNR-Strep network. Invasive group A streptococcal infections in adults, France (2006-2010). Clin Microbiol Infect. 2012;18:702-10. http://dx.doi.org/10.1111/ j.1469-0691.2011.03624.x

12. Clinical and Laboratory Standards Institute. Performance standards for antimicrobial susceptibility testing; twenty-fifth informational supplement (M100-S25). Wayne (PA): The Institute; 2015.

13. Metzgar D, Zampolli A. The M protein of group A Streptococcus is a key virulence factor and a clinically relevant strain identification marker. Virulence. 2011;2:402-12. http://dx.doi.org/ 10.4161/viru.2.5.16342

14. Mihaila-Amrouche L, Bouvet A, Loubinoux J. Clonal spread of emm type 28 isolates of Streptococcus pyogenes that are multiresistant to antibiotics. J Clin Microbiol. 2004;42:3844-6. http://dx.doi.org/10.1128/JCM.42.8.3844-3846.2004

15. Davies MR, Holden MT, Coupland P, Chen JH, Venturini C, Barnett TC, et al. Emergence of scarlet fever Streptococcus pyogenes emm 12 clones in Hong Kong is associated with toxin acquisition and multidrug resistance. Nat Genet. 2015;47:84-7. http://dx.doi.org/10.1038/ng.3147

Address for correspondence: Jae Keun Chung, 149, Hwajeong-ro, Seogu, Gwangju, Korea 61986; email: jkchung@korea.kr; Yeongjin Hong, 322, Seoyang-ro, Hwasun-eup. Hwasun-gun Jeonnam, South Korea 58128; email: yjhong@chonnam.ac.kr 